\title{
Hierarchical Convergence of a Double-Net Algorithm for Equilibrium Problems and Variational Inequality Problems
}

\author{
Yonghong Yao, ${ }^{1}$ Yeong-Cheng Liou, ${ }^{2}$ and Chia-Ping Chen ${ }^{3}$ \\ ${ }^{1}$ Department of Mathematics, Tianjin Polytechnic University, Tianjin 300160, China \\ ${ }^{2}$ Department of Information Management, Cheng Shiu University, Kaohsiung 833, Taiwan \\ ${ }^{3}$ Department of Computer Science and Engineering, National Sun Yat-sen University, \\ Kaohsiung 80424, Taiwan \\ Correspondence should be addressed to Chia-Ping Chen, cpchen@cse.nsysu.edu.tw
}

Received 21 May 2010; Accepted 22 December 2010

Academic Editor: Satit Saejung

Copyright (c) 2010 Yonghong Yao et al. This is an open access article distributed under the Creative Commons Attribution License, which permits unrestricted use, distribution, and reproduction in any medium, provided the original work is properly cited.

\begin{abstract}
We consider the following hierarchical equilibrium problem and variational inequality problem (abbreviated as HEVP): find a point $x^{*} \in \operatorname{EP}(F, B)$ such that $\left\langle A x^{*}, x-x^{*}\right\rangle \geq 0$, for all $x \in$ $\mathrm{EP}(F, B)$, where $A, B$ are two monotone operators and $\operatorname{EP}(F, B)$ is the solution of the equilibrium problem of finding $z \in C$ such that $F(z, y)+\langle B z, y-z\rangle \geq 0$, for all $y \in C$. We note that the problem (HEVP) includes some problems, for example, mathematical program and hierarchical minimization problems as special cases. For solving (HEVP), we propose a double-net algorithm which generates a net $\left\{x_{s, t}\right\}$. We prove that the net $\left\{x_{s, t}\right\}$ hierarchically converges to the solution of (HEVP); that is, for each fixed $t \in(0,1)$, the net $\left\{x_{s, t}\right\}$ converges in norm, as $s \rightarrow 0$, to a solution $x_{t} \in \mathrm{EP}(F, B)$ of the equilibrium problem, and as $t \rightarrow 0$, the net $\left\{x_{t}\right\}$ converges in norm to the unique solution $x^{*}$ of (HEVP).
\end{abstract}

\section{Introduction}

Let $H$ be a real Hilbert space with inner product $\langle\cdot, \cdot\rangle$ and norm $\|\cdot\|$, respectively, and let $C$ be a nonempty closed convex subset of $H$. Recall that a mapping $A$ of $C$ into $H$ is called monotone if

$$
\langle A u-A v, u-v\rangle \geq 0,
$$

for all $u, v \in C$ and $A: C \rightarrow H$ is called $\alpha$-inverse strongly monotone mapping if there exists a positive real number $\alpha$ such that

$$
\langle A u-A v, u-v\rangle \geq \alpha\|A u-A v\|^{2},
$$


for all $u, v \in C$. It is obvious that any $\alpha$-inverse strongly monotone mapping $A$ is monotone and $1 / \alpha$-Lipschitz continuous.

Recently, the following problem has attracted much attention: find hierarchically a fixed point of a nonexpansive mapping $T$ with respect to a nonexpansive mapping $P$, namely,

Find $\tilde{x} \in \operatorname{Fix}(T)$ such that $\langle\tilde{x}-P \tilde{x}, \tilde{x}-x\rangle \leq 0, \quad \forall x \in \operatorname{Fix}(T)$.

Some algorithms for solving the hierarchical fixed point problem (1.3) have been introduced by many authors. For related works, please see, for instance, [1-9] and the references therein.

Remark 1.1. It is not hard to check that solving (1.3) is equivalent to the fixed point problem

$$
\text { Find } \tilde{x} \in C \text { such that } \tilde{x}=\operatorname{proj}_{\mathrm{Fix}(T)} \cdot P \tilde{x} \text {, }
$$

where $\operatorname{proj}_{\mathrm{Fix}(T)}$ stands for the metric projection on the closed convex set Fix $(T)$. By using the definition of the normal cone to $\operatorname{Fix}(T)$, that is,

$$
N_{\mathrm{Fix}(T)}: x \longmapsto \begin{cases}\{u \in H \mid\langle u, y-x\rangle \leq 0, \forall y \in \operatorname{Fix}(T)\} & \text { if } x \in \operatorname{Fix}(T) \\ \emptyset, & \text { otherwise }\end{cases}
$$

we easily prove that (1.3) is equivalent to the variational inequality

$$
0 \in(I-P) \tilde{x}+N_{\operatorname{Fix}(T)} \tilde{x}
$$

At this point, we wish to point out the link with some monotone variational inequalities and convex programming problems as follows.

Example 1.2. Setting $P=I-\gamma A$, where $A$ is $\eta$-Lipschitzian and $k$-strongly monotone with $\gamma \in\left(0,2 k / \eta^{2}\right]$, then $(1.3)$ reduces to

Find $\tilde{x} \in \operatorname{Fix}(T)$ such that $\langle A \tilde{x}, x-\tilde{x}\rangle \geq 0, \quad \forall x \in \operatorname{Fix}(T)$,

a variational inequality studied by Yamada and Ogura [10].

Example 1.3. Let $A$ be a maximal monotone operator. Taking $T=J_{\lambda}^{A}:=(I+\lambda A)^{-1}$ and $P=$ $I-\gamma \nabla \psi$, where $\psi$ is a convex function such that $\nabla \psi$ is $\eta$-Lipschitzian (which is equivalent to the fact that $\nabla \psi$ is $\eta^{-1}$ cocoercive) with $\gamma \in(0,2 / \eta]$, and $\operatorname{Fix}\left(J_{\lambda}^{A}\right)=A^{-1}(0)$. Then (1.3) reduces 
to the following mathematical program with generalized equation constraint:

$$
\min _{0 \in A(x)} \psi(x)
$$

a problem considered by Luo et al. [11].

Example 1.4. Taking $A=\partial \varphi$, where $\partial \varphi$ is the subdifferential of a lower semicontinuous convex function, then (1.8) reduces to the following hierarchical minimization problem considered in Cabot [12] and Solodov [13]:

$$
\min _{x \in \arg \min \varphi} \psi(x)
$$

Let $B: C \rightarrow H$ be a nonlinear mapping, and let $F$ be a bifunction of $C \times C$ into $\mathbf{R}$. Consider the following equilibrium problem of finding $z \in C$ such that

$$
F(z, y)+\langle B z, y-z\rangle \geq 0, \quad \forall y \in C
$$

If $B=0$, then (1.10) reduces to

$$
F(z, y) \geq 0, \quad \forall y \in C
$$

The solution set of equilibrium problems (1.10) and (1.11) are denoted by $\operatorname{EP}(F, B)$ and $\operatorname{EP}(F)$, respectively. The equilibrium problem (1.10) is very general in the sense that it includes, as special cases, optimization problems, variational inequalities, fixed point problems, minimax problems, Nash equilibrium problem in noncooperative games, and others. We remind the readers to refer to [14-30] and the references therein.

Motivated and inspired by the above works, in this paper, we consider the following hierarchical equilibrium problem and variational inequality problem: find a point $x^{*} \in$ $\mathrm{EP}(F, B)$ such that

$$
\left\langle A x^{*}, x-x^{*}\right\rangle \geq 0, \quad \forall x \in \mathrm{EP}(F, B),
$$

where $A, B$ are two monotone operators. The solution set of (1.12) is denoted by $\Omega$.

Remark 1.5. It is clear that the hierarchical variational inequality problem and equilibrium problem (1.12) includes the variational inequality problem studied by Yamada and Ogura [10], mathematical program studied by Luo et al. [11], hierarchical minimization problem considered by Cabot [12] and Solodov [13], as special cases.

For solving (1.12), we propose a double-net algorithm which generates a net $\left\{x_{s, t}\right\}$. We prove that the net $\left\{x_{s, t}\right\}$ hierarchically converges to the solution of (1.12); that is, for each fixed $t \in(0,1)$, the net $\left\{x_{s, t}\right\}$ converges in norm, as $s \rightarrow 0$, to a solution $x_{t} \in \operatorname{EP}(F, B)$ of the equilibrium problem, and as $t \rightarrow 0$, the net $\left\{x_{t}\right\}$ converges in norm to the unique solution $x^{*} \in \Omega$ of (1.12). 


\section{Preliminaries}

Let $H$ be a real Hilbert space. Throughout this paper, let us assume that a bifunction $F$ : $H \times H \rightarrow \mathbf{R}$ satisfies the following conditions:

(F1) $F(x, x)=0$ for all $x \in H$;

(F2) $F$ is monotone, that is, $F(x, y)+F(y, x) \leq 0$ for all $x, y \in H$;

(F3) for each $x, y, z \in H, \lim \sup _{t \backslash 0} F(t z+(1-t) x, y) \leq F(x, y)$;

(F4) for each $x \in H, y \mapsto F(x, y)$ is convex and lower semicontinuous.

On the equilibrium problems, we have the following important lemma. You can find it in [31].

Lemma 2.1. Let $H$ be a real Hilbert space, and let $F$ be a bifunction of $H \times H$ into $R$ satisfying conditions (F1)-(F4). Let $r>0$, and $x \in H$. Then, there exists $z \in H$ such that

$$
F(z, y)+\frac{1}{r}\langle y-z, z-x\rangle \geq 0, \quad \forall y \in H
$$

Further, if $T_{r}(x)=\{z \in H \mid F(z, y)+(1 / r)\langle y-z, z-x\rangle \geq 0$, for all $y \in H\}$, then the following hold:

(1) $T_{r}$ is single-valued;

(2) $T_{r}$ is firmly nonexpansive; that is, for any $x, y \in H$,

$$
\left\|T_{r} x-T_{r} y\right\|^{2} \leq\left\langle T_{r} x-T_{r} y, x-y\right\rangle
$$

(3) $\operatorname{Fix}\left(T_{r}\right)=\operatorname{EP}(F)$;

(4) $\mathrm{EP}(F)$ is closed and convex. sections.

Below we gather some basic facts that are needed in the argument of the subsequent

Lemma 2.2 (see [32]). Let $H$ be a real Hilbert space. Let the mapping $A: H \rightarrow H$ be $\alpha$-inverse strongly monotone, and let $\lambda>0$ be a constant. Then, one has

$$
\|(I-\lambda A) x-(I-\lambda A) y\|^{2} \leq\|x-y\|^{2}+\lambda(\lambda-2 \alpha)\|A x-A y\|^{2}, \quad \forall x, y \in H .
$$

In particular, if $0 \leq \lambda \leq 2 \alpha$, then $I-\lambda A$ is nonexpansive.

Lemma 2.3 (demiclosedness principle for nonexpansive mappings, see [33]). Let $C$ be a nonempty closed convex subset of a real Hilbert space $H$ and let $T: C \rightarrow C$ be a nonexpansive mapping with $\operatorname{Fix}(T) \neq \emptyset$. If $\left\{x_{n}\right\}$ is a sequence in $C$ weakly converging to $x$, and if $\left\{(I-T) x_{n}\right\}$ converges strongly to $y$, then $(I-T) x=y$; in particular, if $y=0$, then $x \in \operatorname{Fix}(T)$. 
Lemma 2.4. Let $H$ be a real Hilbert space. Let $f: H \rightarrow H$ be a $\rho$-contraction with coefficient $\rho \in[0,1)$. Let the mapping $A: H \rightarrow H$ be $\alpha$-inverse strongly monotone. Let $\lambda \in(0,2 \alpha)$, and $t \in(0,1)$. Then the variational inequality

$$
x^{*} \in \operatorname{EP}(F, B), \quad\left\langle t f(z)+(1-t)(I-\lambda A) z-z, x^{*}-z\right\rangle \geq 0, \quad \forall z \in \operatorname{EP}(F, B)
$$

is equivalent to the dual variational inequality

$$
x^{*} \in \operatorname{EP}(F, B), \quad\left\langle t f\left(x^{*}\right)+(1-t)(I-\lambda A) x^{*}-x^{*}, x^{*}-z\right\rangle \geq 0, \quad \forall z \in \operatorname{EP}(F, B) .
$$

Proof. Assume that $x^{*} \in \mathrm{EP}(F, B)$ solves (2.4). For all $z \in \mathrm{EP}(F, B)$, set

$$
x=x^{*}+s\left(z-x^{*}\right) \in \operatorname{EP}(F, B), \quad 0<s<1 .
$$

We note that

$$
\left\langle t f(x)+(1-t)(I-\lambda A) x-x, x^{*}-x\right\rangle \geq 0 .
$$

Hence, we have

$$
\left\langle t f\left(x^{*}+s\left(z-x^{*}\right)\right)+(1-t)(I-\lambda A)\left(x^{*}+s\left(z-x^{*}\right)\right)-x^{*}-s\left(z-x^{*}\right), s\left(x^{*}-z\right)\right\rangle \geq 0,
$$

which implies that

$$
\left\langle t f\left(x^{*}+s\left(z-x^{*}\right)\right)+(1-t)(I-\lambda A)\left(x^{*}+s\left(z-x^{*}\right)\right)-x^{*}-s\left(z-x^{*}\right), x^{*}-z\right\rangle \geq 0 .
$$

Letting $s \rightarrow 0$, we have

$$
\left\langle t f\left(x^{*}\right)+(1-t)(I-\lambda A) x^{*}-x^{*}, x^{*}-z\right\rangle \geq 0,
$$

which is exactly (2.5).

Assume that $x^{*}$ solves (2.5). Hence,

$$
\left\langle t f\left(x^{*}\right)+(1-t)(I-\lambda A) x^{*}-x^{*}, x^{*}-z\right\rangle \geq 0 .
$$

Noting that $I-f$ and $A$ are monotone, we have

$$
\begin{gathered}
\left\langle(I-f) z-(I-f) x^{*}, z-x^{*}\right\rangle \geq 0 \\
\left\langle A z-A x^{*}, z-x^{*}\right\rangle \geq 0
\end{gathered}
$$

It follows that

$$
t\left\langle(I-f) z-(I-f) x^{*}, z-x^{*}\right\rangle+(1-t) \lambda\left\langle A z-A x^{*}, z-x^{*}\right\rangle \geq 0,
$$


which implies that

$$
\left\langle t f(z)+(1-t)(I-\lambda A) z-z, x^{*}-z\right\rangle \geq\left\langle t f\left(x^{*}\right)+(1-t)(I-\lambda A) x^{*}-x^{*}, x^{*}-z\right\rangle \geq 0 .
$$

This implies that $x^{*}$ solves (2.4). The proof is completed.

\section{Main Results}

In this section, we first introduce our double-net algorithm.

Let $H$ be a real Hilbert space. Let $f: H \rightarrow H$ be a $\rho$-contraction with coefficient $\rho \in[0,1)$. Let the mappings $A, B: H \rightarrow H$ be $\alpha$-inverse strongly monotone and $\beta$-inverse strongly monotone, respectively. Let $F$ be a bifunction from $H \times H \rightarrow \mathbf{R}$, and let $\lambda \in(0,2 \alpha)$ and $r \in(0,2 \beta)$ be two constants. For $s, t \in(0,1)$, we define the following mapping:

$$
x \longmapsto W_{s, t} x:=s[t f(x)+(1-t)(x-\lambda A x)]+(1-s) T_{r}(x-r B x),
$$

where $T_{r}(x)$ is defined by Lemma 2.1. We note that the mapping $W_{s, t}$ is a contraction. As a matter of fact, we have

$$
\begin{aligned}
\left\|W_{s, t} x-W_{s, t} y\right\|= & \| s[t f(x)+(1-t)(x-\lambda A x)]+(1-s) T_{r}(x-r B x) \\
& \quad-s[t f(y)+(1-t)(y-\lambda A y)]-(1-s) T_{r}(y-r B y) \| \\
\leq & s t\|f(x)-f(y)\|+s(1-t)\|(x-\lambda A x)-(y-\lambda A y)\| \\
& +(1-s)\left\|T_{r}(x-r B x)-T_{r}(y-r B y)\right\| \\
\leq & s t \rho\|x-y\|+s(1-t)\|x-y\|+(1-s)\|x-y\| \\
= & {[1-(1-\rho) s t]\|x-y\|, }
\end{aligned}
$$

which implies that the mapping $W_{s, t}$ is contractive. Hence, by Banach's contraction principle, $W_{s, t}$ has a unique fixed point which is denoted $x_{s, t} \in H$; that is, $x_{s, t}$ is the unique solution in $H$ of the fixed point equation

$$
x_{s, t}=s\left[t f\left(x_{s, t}\right)+(1-t)\left(x_{s, t}-\lambda A x_{s, t}\right)\right]+(1-s) T_{r}\left(x_{s, t}-r B x_{s, t}\right), \quad s, t \in(0,1) .
$$

Below is our main result of this paper which displays the behavior of the net $\left\{x_{s, t}\right\}$ as $s \rightarrow 0$ and $t \rightarrow 0$ successively.

Theorem 3.1. Let $H$ be a real Hilbert space. Let $f: H \rightarrow H$ be a $\rho$-contraction with coefficient $\rho \in[0,1)$. Let the mappings $A, B: H \rightarrow H$ be $\alpha$-inverse strongly monotone and $\beta$-inverse strongly monotone, respectively. Let $\lambda \in(0,2 \alpha)$ and $r \in(0,2 \beta)$ be two constants. Let $F$ be a bifunction from $H \times H \rightarrow R$ satisfying (F1)-(F4). Suppose the solution set $\Omega$ of (1.12) is nonempty. Let, for each $(s, t) \in(0,1)^{2}, x_{s, t}$ be defined implicitly by (3.3). Then, the net $\left\{x_{s, t}\right\}$ hierarchically converges to the unique solution $x^{*}$ of the hierarchical equilibrium problem and variational inequality problem (1.12). That is to say, for each fixed $t \in(0,1)$, the net $\left\{x_{s, t}\right\}$ converges in norm, as $s \rightarrow 0$, to a solution 
$x_{t} \in \mathrm{EP}(F, B)$ of the equilibrium problem (1.10). Moreover, as $t \rightarrow 0$, the net $\left\{x_{t}\right\}$ converges in norm to the unique solution $x^{*} \in \Omega$. Furthermore, $x^{*}$ also solves the following variational inequality:

$$
x^{*} \in \Omega, \quad\left\langle(I-f) x^{*}, x-x^{*}\right\rangle \geq 0, \quad \forall x \in \Omega
$$

We divide our detailed proofs into several conclusions as follows. Throughout, we assume all assumptions of Theorem 3.1 are satisfied.

Conclusion 1. For each fixed $t \in(0,1)$, the net $\left\{x_{s, t}\right\}$ is bounded.

Proof. Take any $z \in \operatorname{EP}(F, B)$. It is clear that $z=T_{r}(z-r B z)$. Set $u_{s, t}=T_{r}\left(x_{s, t}-r B x_{s, t}\right)$ for all $s, t \in(0,1)$. Since $T_{r}, I-\lambda A$ and $I-r B$ are nonexpansive (by Lemmas 2.1 and 2.2), we have from (3.3) that

$$
\begin{aligned}
\left\|x_{s, t}-z\right\|= & \left\|s\left[t f\left(x_{s, t}\right)+(1-t)(I-\lambda A) x_{s, t}\right]+(1-s) T_{r}\left(x_{s, t}-r B x_{s, t}\right)-z\right\| \\
\leq & s\left\|t f\left(x_{s, t}\right)+(1-t)(I-\lambda A) x_{s, t}-z\right\|+(1-s)\left\|T_{r}\left(x_{s, t}-r B x_{s, t}\right)-T_{r}(z-r B z)\right\| \\
\leq & s\left[t\left\|f\left(x_{s, t}\right)-f(z)\right\|+t\|f(z)-z\|\right. \\
& \left.\quad+(1-t)\left\|(I-\lambda A) x_{s, t}-(I-\lambda A) z\right\|+(1-t)\|(I-\lambda A) z-z\|\right]+(1-s)\left\|x_{s, t}-z\right\| \\
\leq & s\left[t \rho\left\|x_{s, t}-z\right\|+t\|f(z)-z\|+(1-t)\left\|x_{s, t}-z\right\|+(1-t) \lambda\|A z\|\right]+(1-s)\left\|x_{s, t}-z\right\| \\
= & {[1-(1-\rho) s t]\left\|x_{s, t}-z\right\|+s t\|f(z)-z\|+s(1-t) \lambda\|A z\| . }
\end{aligned}
$$

This implies that

$$
\begin{aligned}
\left\|x_{s, t}-z\right\| & \leq \frac{1}{(1-\rho) t}(t\|f(z)-z\|+(1-t) \lambda\|A z\|) \\
& \leq \frac{1}{(1-\rho) t} \max \{\|f(z)-z\|, \lambda\|A z\|\}
\end{aligned}
$$

It follows that for each fixed $t \in(0,1),\left\{x_{s, t}\right\}$ is bounded, so are the nets $\left\{f\left(x_{s, t}\right)\right\},\left\{(I-\lambda A) x_{s, t}\right\}$ and $\left\{u_{s, t}\right\}$. Note that we use $M_{t}$ as a positive constant which bounds all bounded terms appearing in the following.

Conclusion 2. $x_{s, t} \rightarrow x_{t} \in \mathrm{EP}(F, B)$ as $s \rightarrow 0$.

Proof. From Lemma 2.2, we have

$$
\begin{aligned}
\left\|x_{s, t}-\lambda A x_{s, t}-(z-\lambda A z)\right\|^{2} & \leq\left\|x_{s, t}-z\right\|^{2}+\lambda(\lambda-2 \alpha)\left\|A x_{s, t}-A z\right\|^{2}, \\
\left\|u_{s, t}-z\right\|^{2} & =\left\|T_{r}\left(x_{s, t}-r B x_{s, t}\right)-T_{r}(z-r B z)\right\|^{2} \\
& \leq\left\|x_{s, t}-r B x_{s, t}-(z-r B z)\right\|^{2} \\
& \leq\left\|x_{s, t}-z\right\|^{2}+r(r-2 \beta)\left\|B x_{s, t}-B z\right\|^{2} .
\end{aligned}
$$


By (3.3), we have

$$
\begin{aligned}
\left\|x_{s, t}-z\right\|^{2}= & s t\left\langle f\left(x_{s, t}\right)-f(z), x_{s, t}-z\right\rangle+s t\left\langle f(z)-z, x_{s, t}-z\right\rangle \\
& +s(1-t)\left\langle(I-\lambda A) x_{s, t}-(I-\lambda A) z, x_{s, t}-z\right\rangle \\
& +s(1-t)\left\langle(I-\lambda A) z-z, x_{s, t}-z\right\rangle \\
& +(1-s)\left\langle T_{r}\left(x_{s, t}-r B x_{s, t}\right)-T_{r}(z-r B z), x_{s, t}-z\right\rangle \\
\leq & s t\left\|f\left(x_{s, t}\right)-f(z)\right\|\left\|x_{s, t}-z\right\|+s t\left\langle f(z)-z, x_{s, t}-z\right\rangle \\
& +s(1-t)\left\|(I-\lambda A) x_{s, t}-(I-\lambda A) z\right\|\left\|x_{s, t}-z\right\|-s(1-t) \lambda\left\langle A z, x_{s, t}-z\right\rangle \\
& +(1-s)\left\|T_{r}\left(x_{s, t}-r B x_{s, t}\right)-T_{r}(z-r B z)\right\|\left\|x_{s, t}-z\right\| \\
\leq & s t \rho\left\|x_{s, t}-z\right\|^{2}+s t\left\langle f(z)-z, x_{s, t}-z\right\rangle-s(1-t) \lambda\left\langle A z, x_{s, t}-z\right\rangle \\
& +s(1-t)\left\|(I-\lambda A) x_{s, t}-(I-\lambda A) z\right\|\left\|x_{s, t}-z\right\| \\
& +(1-s)\left\|(I-r B) x_{s, t}-(I-r B) z\right\|\left\|x_{s, t}-z\right\| \\
\leq & s t \rho\left\|x_{s, t}-z\right\|^{2}+s t\left\langle f(z)-z, x_{s, t}-z\right\rangle-s(1-t) \lambda\left\langle A z, x_{s, t}-z\right\rangle \\
& +\frac{s(1-t)}{2}\left(\left\|(I-\lambda A) x_{s, t}-(I-\lambda A) z\right\|^{2}+\left\|x_{s, t}-z\right\|^{2}\right) \\
& +\frac{1-s}{2}\left(\left\|(I-r B) x_{s, t}-(I-r B) z\right\|^{2}+\left\|x_{s, t}-z\right\|^{2}\right) .
\end{aligned}
$$

This together with (3.7) implies that

$$
\begin{aligned}
\left\|x_{s, t}-z\right\|^{2} \leq & s t \rho\left\|x_{s, t}-z\right\|^{2}+s t\left\langle f(z)-z, x_{s, t}-z\right\rangle-s(1-t) \lambda\left\langle A z, x_{s, t}-z\right\rangle \\
& +\frac{s(1-t)}{2}\left(\left\|x_{s, t}-z\right\|^{2}+\lambda(\lambda-2 \alpha)\left\|A x_{s, t}-A z\right\|^{2}+\left\|x_{s, t}-z\right\|^{2}\right) \\
& +\frac{1-s}{2}\left(\left\|x_{s, t}-z\right\|^{2}+r(r-2 \beta)\left\|B x_{s, t}-B z\right\|^{2}+\left\|x_{s, t}-z\right\|^{2}\right) \\
= & {[1-(1-\rho) s t]\left\|x_{s, t}-z\right\|^{2}+s t\left\langle f(z)-z, x_{s, t}-z\right\rangle-s(1-t) \lambda\left\langle A z, x_{s, t}-z\right\rangle } \\
& +\frac{s(1-t)}{2} \lambda(\lambda-2 \alpha)\left\|A x_{s, t}-A z\right\|^{2}+\frac{1-s}{2} r(r-2 \beta)\left\|B x_{s, t}-B z\right\|^{2} .
\end{aligned}
$$

It follows that

$$
\begin{aligned}
& (1-s) r(2 \beta-r)\left\|B x_{s, t}-B z\right\|^{2} \\
& \leq-2(1-\rho) s t\left\|x_{s, t}-z\right\|^{2}+2 s t\|f(z)-z\|\left\|x_{s, t}-z\right\| \\
& \quad-2 s(1-t) \lambda\|A z\|\left\|x_{s, t}-z\right\|+s(1-t) \lambda(\lambda-2 \alpha)\left\|A x_{s, t}-A z\right\|^{2} \\
& \longrightarrow 0 \quad \text { as } s \longrightarrow 0 \text { for each fixed } t \in(0,1) .
\end{aligned}
$$


Therefore

$$
\lim _{s \rightarrow 0}\left\|B x_{s, t}-B z\right\|=0
$$

Using Lemma 2.1, we obtain

$$
\begin{aligned}
\left\|u_{s, t}-z\right\|^{2}= & \left\|T_{r}\left(x_{s, t}-r B x_{s, t}\right)-T_{r}(z-r B z)\right\|^{2} \\
\leq & \left\langle\left(x_{s, t}-r B x_{s, t}\right)-(z-r B z), u_{s, t}-z\right\rangle \\
= & \frac{1}{2}\left(\left\|\left(x_{s, t}-r B x_{s, t}\right)-(z-r B z)\right\|^{2}+\left\|u_{s, t}-z\right\|^{2}\right. \\
& \left.\quad-\left\|\left(x_{s, t}-z\right)-r\left(B x_{s, t}-B z\right)-\left(u_{s, t}-z\right)\right\|^{2}\right) \\
\leq & \frac{1}{2}\left(\left\|x_{s, t}-z\right\|^{2}+\left\|u_{s, t}-z\right\|^{2}-\left\|\left(x_{s, t}-u_{s, t}\right)-r\left(B x_{s, t}-B z\right)\right\|^{2}\right) \\
= & \frac{1}{2}\left(\left\|x_{s, t}-z\right\|^{2}+\left\|u_{s, t}-z\right\|^{2}-\left\|x_{s, t}-u_{s, t}\right\|^{2}\right. \\
& \left.+2 r\left\langle x_{s, t}-u_{s, t}, B x_{s, t}-B z\right\rangle-r^{2}\left\|B x_{s, t}-B z\right\|^{2}\right),
\end{aligned}
$$

which implies that

$$
\begin{aligned}
\left\|u_{s, t}-z\right\|^{2} & \leq\left\|x_{s, t}-z\right\|^{2}-\left\|x_{s, t}-u_{s, t}\right\|^{2}+2 r\left\langle x_{s, t}-u_{s, t}, B x_{s, t}-B z\right\rangle-r^{2}\left\|B x_{s, t}-B z\right\|^{2} \\
& \leq\left\|x_{s, t}-z\right\|^{2}-\left\|x_{s, t}-u_{s, t}\right\|^{2}+2 r\left\|x_{s, t}-u_{s, t}\right\|\left\|B x_{s, t}-B z\right\| .
\end{aligned}
$$

From (3.3), we have

$$
\begin{aligned}
\left\|x_{s, t}-z\right\| & =\left\|(1-s)\left(u_{s, t}-z\right)+s\left[t f\left(x_{s, t}\right)+(1-t)\left(x_{s, t}-\lambda A x_{s, t}\right)-z\right]\right\| \\
& \leq\left\|u_{s, t}-z\right\|+s M_{t} .
\end{aligned}
$$

Hence,

$$
\begin{aligned}
\left\|x_{s, t}-z\right\|^{2} & \leq\left\|u_{s, t}-z\right\|^{2}+s M_{t} \\
& \leq\left\|x_{s, t}-z\right\|^{2}-\left\|x_{s, t}-u_{s, t}\right\|^{2}+M_{t}\left\|B x_{s, t}-B z\right\|+s M_{t} .
\end{aligned}
$$

It follows that

$$
\left\|x_{s, t}-u_{s, t}\right\|^{2} \leq M_{t}\left\|B x_{s, t}-B z\right\|+s M_{t} \longrightarrow 0 \quad \text { as } s \longrightarrow 0 \text { for each fixed } t \in(0,1)
$$


Next, we show that, for each fixed $t \in(0,1)$, the net $\left\{x_{s, t}\right\}$ is relatively norm-compact as $s \rightarrow 0$. It follows from (3.8) that

$$
\begin{aligned}
\left\|x_{s, t}-z\right\|^{2}= & s t\left\langle f\left(x_{s, t}\right)-f(z), x_{s, t}-z\right\rangle+s t\left\langle f(z)-z, x_{s, t}-z\right\rangle \\
& +s(1-t)\left\langle(I-\lambda A) x_{s, t}-(I-\lambda A) z, x_{s, t}-z\right\rangle \\
& +s(1-t)\left\langle(I-\lambda A) z-z, x_{s, t}-z\right\rangle \\
& +(1-s)\left\langle T_{r}\left(x_{s, t}-r B x_{s, t}\right)-T_{r}(z-r B z), x_{s, t}-z\right\rangle \\
\leq & s t \rho\left\|x_{s, t}-z\right\|^{2}+s t\left\langle f(z)-z, x_{s, t}-z\right\rangle+s(1-t)\left\|x_{s, t}-z\right\|^{2} \\
& +s(1-t)\left\langle(I-\lambda A) z-z, x_{s, t}-z\right\rangle+(1-s)\left\|x_{s, t}-z\right\|^{2} \\
= & {[1-(1-\rho) s t]\left\|x_{s, t}-z\right\|^{2}+s t\left\langle f(z)-z, x_{s, t}-z\right\rangle-s(1-t) \lambda\left\langle A z, x_{s, t}-z\right\rangle . }
\end{aligned}
$$

It turns out that

$$
\left\|x_{s, t}-z\right\|^{2} \leq \frac{1}{(1-\rho) t}\left\langle t f(z)+(1-t)(I-\lambda A) z-z, x_{s, t}-z\right\rangle, \quad z \in \operatorname{EP}(F, B)
$$

Assume that $\left\{s_{n}\right\} \subset(0,1)$ is such that $s_{n} \rightarrow 0$ as $n \rightarrow \infty$. By (3.18), we conclude immediately that

$$
\left\|x_{S_{n}, t}-z\right\|^{2} \leq \frac{1}{(1-\rho) t}\left\langle t f(z)+(1-t)(I-\lambda A) z-z, x_{S_{n}, t}-z\right\rangle, \quad z \in \operatorname{EP}(F, B) .
$$

Since $\left\{x_{s_{n}, t}\right\}$ is bounded, without loss of generality, we may assume that as $s_{n} \rightarrow 0,\left\{x_{s_{n}, t}\right\}$ converges weakly to a point $x_{t}$. Note that $\left\{u_{s_{n}, t}\right\}$ also converges weakly to a point $x_{t}$.

Now we show that $x_{t} \in \mathrm{EP}$. Since $u_{s_{n}, t}=T_{r}\left(x_{s_{n}, t}-r B x_{s_{n}, t}\right)$, for any $y \in H$, we have

$$
F\left(u_{s_{n}, t}, y\right)+\frac{1}{r}\left\langle y-u_{s_{n}, t}, u_{s_{n}, t}-\left(x_{s_{n}, t}-r B x_{s_{n}, t}\right)\right\rangle \geq 0
$$

From the monotonicity of $F$, we have

$$
\frac{1}{r}\left\langle y-u_{s_{n}, t}, u_{s_{n}, t}-\left(x_{s_{n}, t}-r B x_{s_{n}, t}\right)\right\rangle \geq F\left(y, u_{s_{n}, t}\right), \quad \forall y \in H .
$$

Hence,

$$
\left\langle y-u_{S_{n_{i}}, t}, \frac{u_{S_{n_{i}}, t}-x_{S_{n_{i}}, t}}{r}+B x_{S_{n_{i}}, t}\right\rangle \geq F\left(y, u_{S_{n_{i}}, t}\right), \quad \forall y \in H .
$$


Put $z_{k}=k y+(1-k) x_{t}$ for all $k \in(0,1]$ and $y \in H$. From (3.22), we have

$$
\begin{aligned}
\left\langle z_{k}-u_{s_{n_{i}}, t}, B z z_{k}\right\rangle \geq & \left\langle z_{k}-u_{s_{n_{i}},}, B z_{k}\right\rangle-\left\langle z_{k}-u_{s_{n_{i}}, t}, \frac{u_{s_{n_{i}}, t}-x_{S_{n_{i}}, t}}{r}+B x_{S_{n_{i}}, t}\right\rangle+F\left(z_{k}, u_{S_{n_{i}}, t}\right) \\
= & \left\langle z_{k}-u_{s_{n_{i}},}, B z_{k}-B u_{S_{n_{i}}, t}\right\rangle+\left\langle z_{k}-u_{S_{n_{i}}, t}, B u_{s_{n_{i}}, t}-B x_{S_{n_{i}}, t}\right\rangle \\
& -\left\langle z_{k}-u_{s_{n_{i}},}, \frac{u_{s_{n_{i}}, t}-x_{S_{n_{i}}, t}}{r}\right\rangle+F\left(z_{k}, u_{S_{n_{i}}, t}\right) .
\end{aligned}
$$

Note that $\left\|B u_{S_{n_{i}}, t}-B x_{S_{n_{i}}, t}\right\| \leq(1 / \beta)\left\|u_{s_{n_{i}}, t}-x_{S_{n_{i}}, t}\right\| \rightarrow 0$. Further, from monotonicity of $B$, we have $\left\langle z_{k}-u_{s_{n_{i}}, t}, B z_{k}-B u_{s_{n_{i}}, t}\right\rangle \geq 0$. Letting $i \rightarrow \infty$ in (3.23), we have

$$
\left\langle z_{k}-x_{t}, B z_{k}\right\rangle \geq F\left(z_{k}, x_{t}\right)
$$

From (F1), (F4), and (3.24), we also have

$$
\begin{aligned}
0 & =F\left(z_{k}, z_{k}\right) \leq k F\left(z_{k}, y\right)+(1-k) F\left(z_{k}, x_{t}\right) \\
& \leq k F\left(z_{k}, y\right)+(1-k)\left\langle z_{k}-x_{t}, B z_{k}\right\rangle \\
& =k F\left(z_{k}, y\right)+(1-k) k\left\langle y-x_{t}, B z_{k}\right\rangle
\end{aligned}
$$

and hence

$$
0 \leq F\left(z_{k}, y\right)+(1-k)\left\langle B z_{k}, y-x_{t}\right\rangle
$$

Letting $k \rightarrow 0$ in (3.26), we have, for each $y \in H$,

$$
0 \leq F\left(x_{t}, y\right)+\left\langle y-x_{t}, B x_{t}\right\rangle
$$

This implies that $x_{t} \in \mathrm{EP}(F, B)$.

We can then substitute $x_{t}$ for $z$ in (3.19) to get

$$
\left\|x_{s_{n}, t}-x_{t}\right\|^{2} \leq \frac{1}{(1-\rho) t}\left\langle t f\left(x_{t}\right)+(1-t)(I-\lambda A) x_{t}-x_{t}, x_{s_{n}, t}-x_{t}\right\rangle .
$$

Consequently, the weak convergence of $\left\{x_{s_{n}, t}\right\}$ to $x_{t}$ actually implies that $x_{s_{n}, t} \rightarrow x_{t}$ strongly. This has proved the relative norm-compactness of the net $\left\{x_{s, t}\right\}$ as $s \rightarrow 0$.

Now we return to (3.19) and take the limit, as $n \rightarrow \infty$, to get

$$
\left\|x_{t}-z\right\|^{2} \leq \frac{1}{(1-\rho) t}\left\langle t f(z)+(1-t)(I-\lambda A) z-z, x_{t}-z\right\rangle, \quad \forall z \in \operatorname{EP}(F, B) .
$$


In particular, $x_{t}$ solves the following variational inequality:

$$
x_{t} \in \mathrm{EP}(F, B), \quad\left\langle t f(z)+(1-t)(I-\lambda A) z-z, x_{t}-z\right\rangle \geq 0, \quad \forall z \in \operatorname{EP}(F, B),
$$

or the equivalent dual variational inequality (see Lemma 2.4)

$$
x_{t} \in \operatorname{EP}(F, B), \quad\left\langle t f\left(x_{t}\right)+(1-t)(I-\lambda A) x_{t}-x_{t}, x_{t}-z\right\rangle \geq 0, \quad \forall z \in \operatorname{EP}(F, B) .
$$

Notice that (3.31) is equivalent to the fact that $x_{t}=P_{\operatorname{EP}(F, B)}(t f+(1-t)(I-\lambda A)) x_{t}$. That is, $x_{t}$ is the unique element in $\operatorname{EP}(F, B)$ of the contraction $P_{\mathrm{EP}(F, B)}(t f+(1-t)(I-\lambda A))$. Clearly, this is sufficient to conclude that the entire net $\left\{x_{s, t}\right\}$ converges in norm to $x_{t} \in \operatorname{EP}(F, B)$ as $s \rightarrow 0$.

Conclusion 3. The net $\left\{x_{t}\right\}$ is bounded.

Proof. In (3.31), we take any $y \in \Omega$ to deduce

$$
\left\langle t f\left(x_{t}\right)+(1-t)(I-\lambda A) x_{t}-x_{t}, x_{t}-y\right\rangle \geq 0
$$

By virtue of the monotonicity of $A$ and the fact that $y \in \Omega$, we have

$$
\left\langle(I-\lambda A) x_{t}-x_{t}, x_{t}-y\right\rangle \leq\left\langle(I-\lambda A) y-y, x_{t}-y\right\rangle \leq 0 .
$$

It follows from (3.32) and (3.33) that

$$
\left\langle f\left(x_{t}\right)-x_{t}, x_{t}-y\right\rangle \geq 0, \quad \forall y \in \Omega .
$$

Hence,

$$
\begin{aligned}
\left\|x_{t}-y\right\|^{2} & \leq\left\langle x_{t}-y, x_{t}-y\right\rangle+\left\langle f\left(x_{t}\right)-x_{t}, x_{t}-y\right\rangle \\
& =\left\langle f\left(x_{t}\right)-f(y), x_{t}-y\right\rangle+\left\langle f(y)-y, x_{t}-y\right\rangle \\
& \leq \rho\left\|x_{t}-y\right\|^{2}+\left\langle f(y)-y, x_{t}-y\right\rangle .
\end{aligned}
$$

Therefore,

$$
\left\|x_{t}-y\right\|^{2} \leq \frac{1}{1-\rho}\left\langle f(y)-y, x_{t}-y\right\rangle, \quad \forall y \in \Omega
$$

In particular,

$$
\left\|x_{t}-y\right\| \leq \frac{1}{1-\rho}\|f(y)-y\|, \quad \forall t \in(0,1)
$$

which implies that $\left(x_{t}\right)$ is bounded. 
Conclusion 4 . The net $x_{t} \rightarrow x^{*} \in \Omega$ which solves the variational inequality VI (3.4).

Proof. First, we note that the solution of the variational inequality VI (3.4) is unique.

We next prove that $\omega_{w}\left(x_{t}\right) \subset \Omega$; namely, if $\left(t_{n}\right)$ is a null sequence in $(0,1)$ such that $x_{t_{n}} \rightarrow x^{\prime}$ weakly as $n \rightarrow \infty$, then $x^{\prime} \in \Omega$. To see this, we use (3.31) to get

$$
\left\langle\lambda A x_{t}, z-x_{t}\right\rangle \geq \frac{t}{1-t}\left\langle(I-f) x_{t}, x_{t}-z\right\rangle, \quad z \in \operatorname{EP}(F, B) .
$$

However, since $A$ is monotone,

$$
\left\langle A z, z-x_{t}\right\rangle \geq\left\langle A x_{t}, z-x_{t}\right\rangle \text {. }
$$

Combining the last two relations yields

$$
\left\langle\lambda A z, z-x_{t}\right\rangle \geq \frac{t}{1-t}\left\langle(I-f) x_{t}, x_{t}-z\right\rangle, \quad z \in \operatorname{EP}(F, B) .
$$

Letting $t=t_{n} \rightarrow 0$ as $n \rightarrow \infty$ in (3.40), we get

$$
\left\langle A z, z-x^{\prime}\right\rangle \geq 0, \quad z \in \operatorname{EP}(F, B)
$$

which is equivalent to its dual variational inequality

$$
\left\langle A x^{\prime}, z-x^{\prime}\right\rangle \geq 0, \quad z \in \operatorname{EP}(F, B) .
$$

Namely, $x^{\prime}$ is a solution of VI (1.12); hence, $x^{\prime} \in \Omega$.

We further prove that $x^{\prime}=x^{*}$, the unique solution of VI (3.4). As a matter of fact, we have by (3.36)

$$
\left\|x_{t_{n}}-x^{\prime}\right\|^{2} \leq \frac{1}{1-\rho}\left\langle f\left(x^{\prime}\right)-x^{\prime}, x_{t_{n}}-x^{\prime}\right\rangle, \quad x^{\prime} \in \Omega .
$$

Therefore, the weak convergence to $x^{\prime}$ of $\left\{x_{t_{n}}\right\}$ implies that $x_{t_{n}} \rightarrow x^{\prime}$ in norm. Now we can let $t=t_{n} \rightarrow 0$ in (3.36) to get

$$
\left\langle f\left(x^{\prime}\right)-x^{\prime}, y-x^{\prime}\right\rangle \leq 0, \quad \forall y \in \Omega .
$$

It turns out that $x^{\prime} \in \Omega$ solves VI (3.4). By uniqueness, we have $x^{\prime}=x^{*}$. This is sufficient to guarantee that $x_{t} \rightarrow x^{*}$ in norm, as $t \rightarrow 0$. The proof is complete.

Proof. By Conclusions 1-4, the proof of Theorem 3.1 is completed. 
Take $B=0$. Then (1.12) reduces to the following: find a point $x^{*} \in \operatorname{EP}(F)$ such that

$$
\left\langle A x^{*}, x-x^{*}\right\rangle \geq 0, \quad \forall x \in \operatorname{EP}(F) .
$$

The solution of (3.45) is denoted by $\Omega_{1}$.

Corollary 3.2. Let $H$ be a real Hilbert space. Let $f: H \rightarrow H$ be a $\rho$-contraction with coefficient $\rho \in[0,1)$. Let the mapping $A: H \rightarrow H$ be $\alpha$-inverse strongly monotone. Let $\lambda \in(0,2 \alpha)$ be a constant. Let $F$ be a bifunction from $H \times H \rightarrow R$ satisfying (F1)-(F4). Suppose the solution set $\Omega_{1}$ is nonempty. Let, for each $(s, t) \in(0,1)^{2}, x_{s, t}$ be defined implicitly by

$$
x_{s, t}=s\left[t f\left(x_{s, t}\right)+(1-t)\left(x_{s, t}-\lambda A x_{s, t}\right)\right]+(1-s) T_{r}\left(x_{s, t}\right), \quad s, t \in(0,1) .
$$

Then, the net $\left\{x_{s, t}\right\}$ hierarchically converges to the unique solution $x^{*}$ of the hierarchical equilibrium problem and variational inequality problem (3.45). That is to say, for each fixed $t \in(0,1)$, the net $\left\{x_{s, t}\right\}$ converges in norm, as $s \rightarrow 0$, to a solution $x_{t} \in \mathrm{EP}(F)$ of the equilibrium problem (1.11). Moreover, as $t \rightarrow 0$, the net $\left\{x_{t}\right\}$ converges in norm to the unique solution $x^{*} \in \Omega_{1}$. Furthermore, $x^{*}$ solves the following variational inequality:

$$
x^{*} \in \Omega_{1}, \quad\left\langle(I-f) x^{*}, x-x^{*}\right\rangle \geq 0, \quad \forall x \in \Omega_{1} .
$$

Taking $A=0$ in Theorem 3.1, we have the following corollary.

Corollary 3.3. Let $H$ be a real Hilbert space. Let $f: H \rightarrow H$ be a $\rho$-contraction with coefficient $\rho \in[0,1)$. Let the mapping $B: H \rightarrow H$ be $\beta$-inverse strongly monotone. Let $r \in(0,2 \beta)$ be a constant. Let $F$ be a bifunction from $H \times H \rightarrow R$ satisfying (F1)-(F4). Suppose that the solution set $\mathrm{EP}(F, B)$ of $(1.10)$ is nonempty. Let, for each $(s, t) \in(0,1)^{2}, x_{s, t}$ be defined implicitly by

$$
x_{s, t}=s\left[t f\left(x_{s, t}\right)+(1-t) x_{s, t}\right]+(1-s) T_{r}\left(x_{s, t}-r B x_{s, t}\right), \quad s, t \in(0,1) .
$$

Then, the net $\left\{x_{s, t}\right\}$ hierarchically converges to the unique solution $x^{*}$ of the equilibrium problem (1.10). That is to say, for each fixed $t \in(0,1)$, the net $\left\{x_{s, t}\right\}$ converges in norm, as $s \rightarrow 0$, to a solution $x_{t} \in \mathrm{EP}(F, B)$ of the equilibrium problem (1.10). Moreover, as $t \rightarrow 0$, the net $\left\{x_{t}\right\}$ converges in norm to the unique solution $x^{*} \in \mathrm{EP}(F, B)$. Furthermore, $x^{*}$ solves the following variational inequality:

$$
x^{*} \in \mathrm{EP}(F, B), \quad\left\langle(I-f) x^{*}, x-x^{*}\right\rangle \geq 0, \quad \forall x \in \mathrm{EP}(F, B) .
$$

Taking $A=B=0$ in Theorem 3.1, we have the following corollary.

Corollary 3.4. Let $H$ be a real Hilbert space. Let $f: H \rightarrow H$ be a $\rho$-contraction with coefficient $\rho \in[0,1)$. Let $F$ be a bifunction from $H \times H \rightarrow R$ satisfying (F1)-(F4). Suppose the solution set $\mathrm{EP}(F)$ of $(1.11)$ is nonempty. Let, for each $(s, t) \in(0,1)^{2}, x_{s, t}$ be defined implicitly by

$$
x_{s, t}=s\left[t f\left(x_{s, t}\right)+(1-t) x_{s, t}\right]+(1-s) T_{r}\left(x_{s, t}\right), \quad s, t \in(0,1) .
$$


Then, the net $\left\{x_{s, t}\right\}$ hierarchically converges to the unique solution $x^{*}$ of the equilibrium problem (1.11). That is to say, for each fixed $t \in(0,1)$, the net $\left\{x_{s, t}\right\}$ converges in norm, as $s \rightarrow 0$, to a solution $x_{t} \in \mathrm{EP}(F)$ of the equilibrium problem (1.11). Moreover, as $t \rightarrow 0$, the net $\left\{x_{t}\right\}$ converges in norm to the unique solution $x^{*} \in \mathrm{EP}(F)$. Furthermore, $x^{*}$ solves the following variational inequality:

$$
x^{*} \in \mathrm{EP}(F), \quad\left\langle(I-f) x^{*}, x-x^{*}\right\rangle \geq 0, \quad \forall x \in \mathrm{EP}(F) .
$$

\section{Acknowledgment}

The work of the second author was partially supported by the Grant NSC 98-2923-E-110-003MY3 and the work of the third author was partially supported by the Grant NSC 98-2221-E110-064.

\section{References}

[1] P.-E. Maingé and A. Moudafi, "Strong convergence of an iterative method for hierarchical fixed-point problems," Pacific Journal of Optimization, vol. 3, no. 3, pp. 529-538, 2007.

[2] L. C. Ceng and A. Petruşel, "Krasnoselski-Mann iterations for hierarchical fixed point problems for a finite family of nonself mappings in Banach spaces," Journal of Optimization Theory and Applications, vol. 146, no. 3, pp. 617-639, 2010.

[3] A. Moudafi, "Krasnoselski-Mann iteration for hierarchical fixed-point problems," Inverse Problems, vol. 23, no. 4, pp. 1635-1640, 2007.

[4] A. Moudafi and P.-E. Maingé, "Towards viscosity approximations of hierarchical fixed-point problems," Fixed Point Theory and Applications, vol. 2006, Article ID 95453, 10 pages, 2006.

[5] Y. Yao and Y.-C. Liou, "Weak and strong convergence of Krasnoselski-Mann iteration for hierarchical fixed point problems," Inverse Problems, vol. 24, no. 1, Article ID 015015, 8 pages, 2008.

[6] F. Cianciaruso, G. Marino, L. Muglia, and Y. Yao, "On a two-step algorithm for hierarchical fixed point problems and variational inequalities," Journal of Inequalities and Applications, vol. 2009, Article ID 208692, 13 pages, 2009.

[7] F. Cianciaruso, V. Colao, L. Muglia, and H.-K. Xu, “On an implicit hierarchical fixed point approach to variational inequalities," Bulletin of the Australian Mathematical Society, vol. 80, no. 1, pp. 117-124, 2009.

[8] X. Lu, H.-K. Xu, and X. Yin, "Hybrid methods for a class of monotone variational inequalities," Nonlinear Analysis: Theory, Methods E Applications, vol. 71, no. 3-4, pp. 1032-1041, 2009.

[9] Y. Yao, R. Chen, and H.-K. Xu, "Schemes for finding minimum-norm solutions of variational inequalities," Nonlinear Analysis: Theory, Methods \& Applications, vol. 72, no. 7-8, pp. 3447-3456, 2010.

[10] I. Yamada and N. Ogura, "Hybrid steepest descent method for variational inequality problem over the fixed point set of certain quasi-nonexpansive mappings," Numerical Functional Analysis and Optimization, vol. 25, no. 7-8, pp. 619-655, 2004.

[11] Z.-Q. Luo, J.-S. Pang, and D. Ralph, Mathematical Programs with Equilibrium Constraints, Cambridge University Press, Cambridge, UK, 1996.

[12] A. Cabot, "Proximal point algorithm controlled by a slowly vanishing term: applications to hierarchical minimization," SIAM Journal on Optimization, vol. 15, no. 2, pp. 555-572, 2005.

[13] M. Solodov, "An explicit descent method for bilevel convex optimization," Journal of Convex Analysis, vol. 14, no. 2, pp. 227-237, 2007.

[14] L.-C. Ceng, S. Al-Homidan, Q. H. Ansari, and J.-C. Yao, "An iterative scheme for equilibrium problems and fixed point problems of strict pseudo-contraction mappings," Journal of Computational and Applied Mathematics, vol. 223, no. 2, pp. 967-974, 2009.

[15] L.-C. Ceng and J.-C. Yao, "A hybrid iterative scheme for mixed equilibrium problems and fixed point problems," Journal of Computational and Applied Mathematics, vol. 214, no. 1, pp. 186-201, 2008. 
[16] Y. Yao, M. A. Noor, and Y.-C. Liou, “On iterative methods for equilibrium problems," Nonlinear Analysis: Theory, Methods \& Applications, vol. 70, no. 1, pp. 497-509, 2009.

[17] J.-W. Peng and J.-C. Yao, "A new hybrid-extragradient method for generalized mixed equilibrium problems, fixed point problems and variational inequality problems," Taiwanese Journal of Mathematics, vol. 12, no. 6, pp. 1401-1432, 2008.

[18] J.-W. Peng and J.-C. Yao, "Some new iterative algorithms for generalized mixed equilibrium problems with strict pseudo-contractions and monotone mappings," Taiwanese Journal of Mathematics, vol. 13, no. 5, pp. 1537-1582, 2009.

[19] L. C. Ceng, A. Petruşel, and J. C. Yao, "Iterative approaches to solving equilibrium problems and fixed point problems of infinitely many nonexpansive mappings," Journal of Optimization Theory and Applications, vol. 143, no. 1, pp. 37-58, 2009.

[20] Y. Yao, Y.-C. Liou, and J.-C. Yao, "A new hybrid iterative algorithm for fixed-point problems, variational inequality problems, and mixed equilibrium problems," Fixed Point Theory and Applications, vol. 2008, Article ID 417089, 15 pages, 2008.

[21] J.-W. Peng, Y.-C. Liou, and J.-C. Yao, “An iterative algorithm combining viscosity method with parallel method for a generalized equilibrium problem and strict pseudocontractions," Fixed Point Theory and Applications, vol. 2009, Article ID 794178, 21 pages, 2009.

[22] L. C. Ceng, G. Mastroeni, and J. C. Yao, "Hybrid proximal-point methods for common solutions of equilibrium problems and zeros of maximal monotone operators," Journal of Optimization Theory and Applications, vol. 142, no. 3, pp. 431-449, 2009.

[23] Y. Yao, Y.-C. Liou, and J.-C. Yao, "Convergence theorem for equilibrium problems and fixed point problems of infinite family of nonexpansive mappings," Fixed Point Theory and Applications, vol. 2007, Article ID 64363, 12 pages, 2007.

[24] Y. Yao, M. A. Noor, S. Zainab, and Y.-C. Liou, "Mixed equilibrium problems and optimization problems," Journal of Mathematical Analysis and Applications, vol. 354, no. 1, pp. 319-329, 2009.

[25] Y. Yao, Y. J. Cho, and R. Chen, "An iterative algorithm for solving fixed point problems, variational inequality problems and mixed equilibrium problems," Nonlinear Analysis: Theory, Methods $\mathcal{E}$ Applications, vol. 71, no. 7-8, pp. 3363-3373, 2009.

[26] G. Marino, V. Colao, L. Muglia, and Y. Yao, "Krasnoselski-Mann iteration for hierarchical fixed points and equilibrium problem," Bulletin of the Australian Mathematical Society, vol. 79, no. 2, pp. 187-200, 2009.

[27] L.-C. Ceng and J.-C. Yao, “A relaxed extragradient-like method for a generalized mixed equilibrium problem, a general system of generalized equilibria and a fixed point problem," Nonlinear Analysis: Theory, Methods E Applications, vol. 72, no. 3-4, pp. 1922-1937, 2010.

[28] L. C. Zeng, Y. C. Lin, and J. C. Yao, "Iterative schemes for generalized equilibrium problem and two maximal monotone operators," Journal of Inequalities and Applications, vol. 2009, Article ID 896252, 34 pages, 2009.

[29] T. Honda, W. Takahashi, and J.-C. Yao, "Nonexpansive retractions onto closed convex cones in Banach spaces," Taiwanese Journal of Mathematics, vol. 14, no. 3B, pp. 1023-1046, 2010.

[30] L.-C. Zeng, Q. H. Ansari, D. S. Shyu, and J.-C. Yao, "Strong and weak convergence theorems for common solutions of generalized equilibrium problems and zeros of maximal monotone operators," Fixed Point Theory and Applications, vol. 2010, Article ID 590278, 33 pages, 2010.

[31] P. L. Combettes and S. A. Hirstoaga, "Equilibrium programming in Hilbert spaces," Journal of Nonlinear and Convex Analysis, vol. 6, no. 1, pp. 117-136, 2005.

[32] W. Takahashi and M. Toyoda, "Weak convergence theorems for nonexpansive mappings and monotone mappings," Journal of Optimization Theory and Applications, vol. 118, no. 2, pp. 417-428, 2003.

[33] K. Goebel and W. A. Kirk, Topics in Metric Fixed Point Theory, vol. 28 of Cambridge Studies in Advanced Mathematics, Cambridge University Press, Cambridge, UK, 1990. 